\title{
Simultaneous Quasiparticle and Josephson Tunneling in BSCCO-2212 Break Junctions
}

\author{
L. Ozyuzer ${ }^{1,2}$, N. Miyakawa $a^{1,3}$, J. F. Zasadzinski ${ }^{1,4}$, Z. Yusof ${ }^{1,4}$, \\ P. Romano ${ }^{1}$, C. Kendziora ${ }^{5}$, P. Guptasarma ${ }^{1}$, D. G. Hinks ${ }^{1}$, K. E. Gray ${ }^{1}$ \\ ${ }^{1}$ Materials Science Division, Argonne National Laboratory, Argonne IL, USA \\ ${ }^{2}$ Department of Physics, Izmir Institute of Technology, Izmir, Turkey \\ ${ }^{3}$ Department of Applied Physics, Science University of Tokyo, Japan \\ ${ }^{4}$ Illinois Institute of Technology, Chicago IL, USA \\ ${ }^{5}$ Naval Research Laboratory, Washington DC, USA,
}

\begin{abstract}
Tunneling measurements are reported for superconductor-insulator-superconductor (SIS) break junctions on underdoped, optimally-doped, and overdoped single crystals of $\mathrm{Bi}_{2} \mathrm{Sr}_{2} \mathrm{CaCu}_{2} \mathrm{O}_{8+\delta}$ (Bi2212). The junction $I-V$ characteristics exhibit welldefined quasiparticle current jumps at $e V=2 \Delta$ as well as hysteretic Josephson currents. The quasiparticle branch has been analyzed in the framework of $d_{x^{2}-y^{2}}$ ( $d$-wave) superconductivity and indicates that there is preferential tunneling along the lobe directions of the $d$-wave gap. For overdoped Bi-2212 with $T_{c}=62 \mathrm{~K}$, the Josephson current is measured as a function of junction resistance, $R_{n}$, which varied by two orders of magnitude $(1 \mathrm{k} \Omega$ to $100 \mathrm{k} \Omega) . I_{c} R_{n}$ product is proportional to the 0.47 power of $I_{c}$ and displays a maximum of $7.0 \mathrm{mV}$. When the hole doping is decreased from overdoped $\left(T_{c}=62 \mathrm{~K}\right)$ to the underdoped regime $\left(T_{c}=70 \mathrm{~K}\right)$, the average $I_{c} R_{n}$ product increases as does the quasiparticle gap. The maximum $I_{c} R_{n}$ is $\sim 40 \%$ of the $\Delta / e$ at each doping level, with a value as high as $25 \mathrm{mV}$ in underdoped Bi-2212.
\end{abstract}

\section{INTRODUCTION}

The wide energy gap, high critical current density, and high transition temperature are advantages of high $T_{c}$ superconductors (HTS) that make them attractive candidates for superconducting electronics.[1] In the past decade, there has been a world-wide effort toward the fabrication of Josephson tunnel junctions, based on HTS. The major difficulty in tunneling junction fabrication is due to the short coherence lengths and anisotropic nature of the superconducting gap in HTS.[2] Two HTS compounds, $\mathrm{YBa}_{2} \mathrm{Cu}_{3} \mathrm{O}_{7-x}$ and $\mathrm{Bi}_{2} \mathrm{Sr}_{2} \mathrm{CaCu}_{2} \mathrm{O}_{8+\delta}(\mathrm{Bi}-2212)$ have been extensively studied to produce high-quality thin film planar junctions. Furthermore intrinsic Josephson tunnel junctions have been fabricated in $\mathrm{Bi}-2212$ and $\mathrm{Tl}_{2} \mathrm{Ba}_{2} \mathrm{CaCu}_{2} \mathrm{O}_{x}$ single crystals.[3] These are considered

Manuscript received September 14, 1998.

L. Ozyuzer, 630-252-8457, fax 630-252-7777, ozyulut@charlie. iit.edu, http://www.iit.edu/ ozyulut; J. F. Zasadzinski, 630-2524067, John_Zasadzinski@qmgate.anl.gov.

This work was partially supported by U.S. Department of Energy, Division of Basic Energy Sciences-Material Sciences under contract No. W-31-109-ENG-38, and the National Science Foundation, Office of Science and Technology Centers under contract No. DMR 91-20000. as c-axis tunnel junctions between $\mathrm{CuO}_{2}$ planes where other layers act as insulating or semiconducting spacers. Additionally, the anisotropic feature of the HTS cuprates has been used for fabricating grain boundary junctions. [4] Misorientation angle of grains behaves as a barrier because of impedance mismatch. However, grain boundary junctions act as weak links between two superconductors and their properties can be explained by resistive shunted junction model. In our case, superconductor-insulatorsuperconductor (SIS) tunnel junctions are formed by break junction method on Bi-2212, producing tunneling spectroscopies with clear indication of Josephson and quasiparticle tunnel currents simultaneously. In this paper, we will present the SIS break junction tunneling results on overdoped, optimally-doped, and underdoped Bi2212 superconductors.

\section{EXPERIMENT}

Two sets of single crystal Bi-2212 were obtained to perform break junction tunneling. The first set of samples of $\mathrm{Bi}-2212$ were grown in a $20 \%$ oxygen atmosphere by a floating zone technique.[5] The second set were grown by a self flux technique in a strong thermal gradient to stabilize the direction of solidification. Both sets of crystals were well characterized and no significant differences were obtained, in spite of the growth methods that are completely different. Overdoping of single crystal Bi-2212 has been conducted using stainless steel cells sealed with samples immersed in liquid oxygen, as described elsewhere.[6] Underdoped crystals have been obtained by vacuum annealing. [5], [7] The crystals in which tunneling measurements were performed have a $T_{c}=62 \mathrm{~K}$ and $82 \mathrm{~K}$ for overdoped, $95 \mathrm{~K}$ for optimally-doped, and $70 \mathrm{~K}$ for underdoped. Tunneling measurements were done with the apparatus described in [8].

SIS break junctions are obtained in an unconventional manner. After the sample is placed in a measurement system and cooled down to $4.2 \mathrm{~K}$, the contact force between the gold tip and sample is adjusted using a differential micrometer. In the ordinary way, a tip pushes onto the surface of the crystal and a superconductor-insulatornormal metal (SIN) junction is formed between the tip and crystal. While the tip is pushed against the crystal, the $I-V$ curve is continuously monitored until an SIN junction is obtained. Here the insulating barrier is the native surface layer of the crystal. Increasing the force 
of the tip punctures the native layer and establishes an ohmic contact $(\sim 1 \mathrm{ohm})$ between the tip and crystal. For Bi-2212, a piece of the crystal dislodges and mechanically binds to the tip. Relieving pressure separates neighboring $\mathrm{Bi}-\mathrm{O}$ planes in $\mathrm{Bi}-2212$, because they are weakly bounded when compared to $\mathrm{Cu}-\mathrm{O}$ and $\mathrm{Sr}-\mathrm{O}$ layers. Consequently, a SIS tunneling break junction can be formed easily with this HTS cuprate. Furthermore, since this tunnel junction is formed in-situ at $4.2 \mathrm{~K}$ and unexposed to air, the barrier interface is clean and impurity free.

\section{Results And Discussion}

A representative $I-V$ curve of a SIS break junction between two pieces of overdoped Bi-2212 single crystal is shown in Fig. 1. The data is taken at $4.2 \mathrm{~K}$ and the junction exhibits sharp current onsets at $\mathrm{eV} \sim \pm 40 \mathrm{meV}$ which corresponds to $\pm 2 \Delta$ as well as a Josephson current at zero bias. Some of the $I-V$ curves present nonlinearity at high bias voltages as in Fig. 1, which might be due to a barrier effect. In such cases, the junction resistance, $R_{n}$, is estimated from the corresponding tunneling conductance at a bias of $200 \mathrm{meV}$. This is not expected to significantly affect our analysis since we are primarily interested in the magnitude of the junction resistance. The inset in the Fig. 1 is an expanded subgap region near zero bias to clearly show the switching nature of the underdamped Josephson junction. Notice that the sweep direction is from negative to positive bias. The optimallydoped and underdoped single crystals of $\mathrm{Bi}-2212$ also show same qualitative characteristics.[5], [7]

The tunneling conductance is proportional to the DOS of a superconductor and for a SIS junction, the tunneling conductance can be expressed as [9]

$$
\frac{d I}{d V}=c \frac{d}{d V} \int|T|^{2} N_{s}(E) N_{s}(E+e V)[F(E)-F(E+e V)] d E
$$

where $F(E)=\left[1+\exp \left(E / k_{B} T\right)\right]^{-1}$ is the Fermi function, $|T|^{2}$ is the tunneling matrix element, $E$ is the quasiparticle energy relative to the Fermi level, $N_{s}(E)$ is the DOS of the superconductor, and $c$ is a proportionality constant. $|T|^{2}$ is taken as a constant to simplify the calculation which is acceptable near the Fermi level. In this work, the DOS of the superconductor is expressed as

$$
N_{s}(E)=\int d \phi f(\phi) \operatorname{Re}\left\{\frac{E-i \Gamma}{\sqrt{(E-i \Gamma)^{2}-\Delta(\phi)^{2}}}\right\}
$$

Here the addition of smearing parameter $\Gamma$ accounts for lifetime effects. This phenomenological model takes into account two factors. First, results from recent tunneling[10], angle resolved photoemission (ARPES)[11], and tricrystal ring[12] experiments on HTS cuprates suggest a strong case for $d_{x^{2}-y^{2}}$ ( $d$-wave) pairing symmetry. In our simple model, this is included by using an angular dependent gap function given by $\Delta(\phi)=\Delta_{0} \cos (2 \phi)[13]$ where $\phi$ is the polar angle in k-space measured with respect to the $(\pi, 0)$ direction. The resulting DOS shows cusplike feature

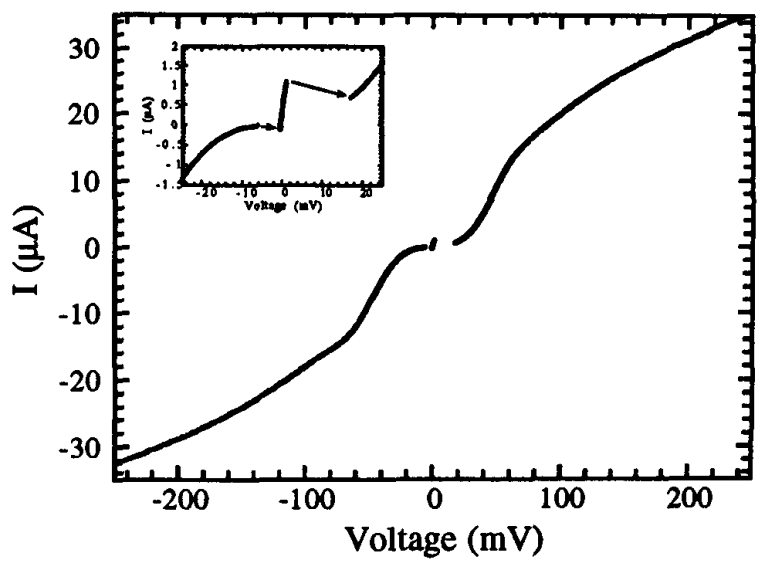

Fig. 1. Current-Voltage characteristics of an overdoped Bi-2212 $\left(T_{c}=62 \mathrm{~K}\right) \mathrm{SIS}$ break junction at $4.2 \mathrm{~K}$. The inset shows the Josephson current in a more sensitive scale.

in the subgap region. However, several tunneling experiments have shown a slight rounding of the subgap region. ARPES results have also suggested that certain particular regions of momentum space are more heavily weighted[11] than others in the contribution to the tunneling current due directly to the band structure. In addition, there may be a certain degree of selectivity in tunneling measurements coming from the tunneling matrix element.[14] For these reasons we have included the second factor in our model which is a weighting function $f(\phi)$ to the DOS model, as is in [10]. Here $f(\phi)=1+\alpha \cos (4 \phi)$ where $\alpha$ is a directionality strength. Although (2) is a phenomenological expression, it will be shown that this weighting function will qualitatively reproduce some of the conductance features observed in the experiment, especially in some SIN junctions. This weighting would also explain the large peak height to background (PHB) ratios observed in SIS junctions as presented in [10].

Fig. 2 shows four representative normalized SIS tunneling conductances for four different doping levels of Bi-2212 (solid lines). Here the Josephson currents have been removed for clarity. The data have been normalized with a third order polynomial. In the figure the dashed lines correspond to numerical fits using our DOS model for $N_{s}(E)$ in (1). There is a good agreement with the experimental data in the subgap region. This means that the experimental results are consistent with the $d$-wave pairing DOS and the suggestion that there is a preferential tunneling along the lobe directions of Bi-2212. It is also observed that the size of the superconducting gap decreases with increasing oxygen doping. However, above the gap region $(\mathrm{eV}>2 \Delta)$ there are significant deviations from experimental results. There are dip and hump features outside the gap region that are also observed in SIN tunneling conductances. These features, which have been suggested to be due to strong coupling effects,[5] cannot be reproduced by our simple model. Fig. 2 also shows that quasiparticle $\mathrm{PHB}$ ratio increases with increasing oxygen doping. It has been suggested by Halbritter[15] that this may be due 


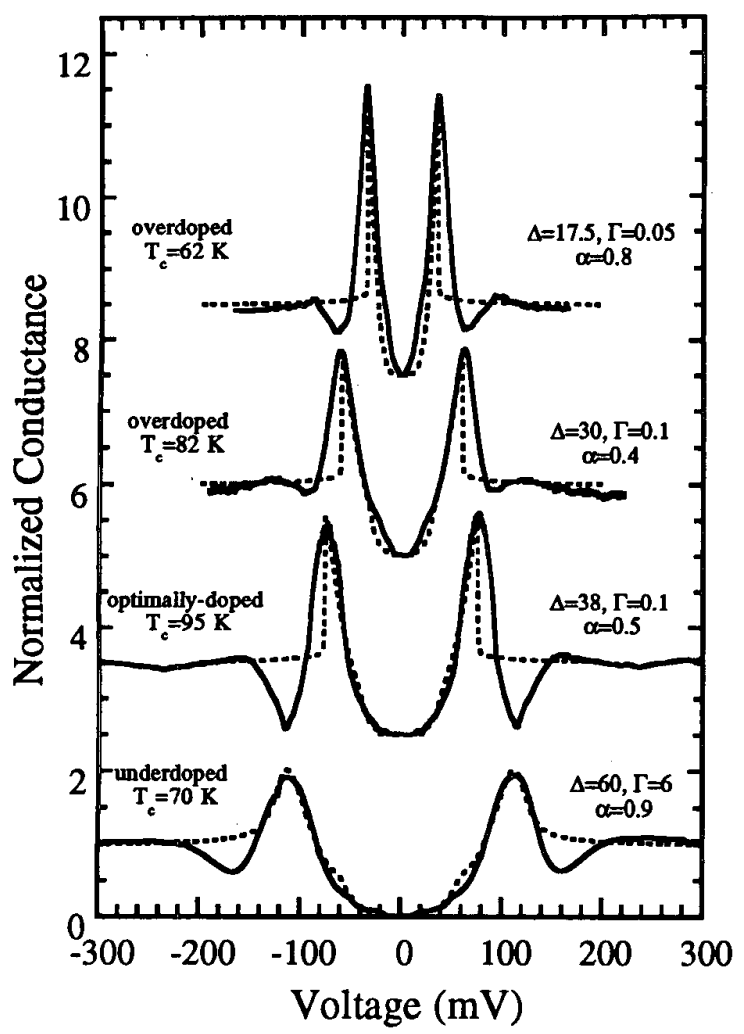

Fig. 2. Normalized conductances of Bi-2212 SIS break junctions for various doping levels. Dashed lines correspond to SIS conductances obtained using the DOS model of (2) with corresponding parameters. For clarity, the data of overdoped $\left(\mathrm{T}_{c}=62 \mathrm{~K}\right)$, overdoped $\left(\mathrm{T}_{c}=82 \mathrm{~K}\right)$ and optimally-doped $\left(\mathrm{T}_{c}=95 \mathrm{~K}\right)$ have been shifted by $7.5,5,2.5$ units respectively.

to the creation of localized states in the barrier region. However, the presence of localized states should manifest itself as additional peaks in the gap region. SIN and SIS tunneling data on overdoped, optimally-doped, and underdoped $\mathrm{Bi}-2212$ do not show these additional peaks. Furthermore, the gap structure disappears at $\mathbf{T}_{c}$ pointing to its superconducting origin. This observation seems to rule out resonant tunneling as the source of the change in PHB ratio with increasing doping. In addition, as clearly seen in Fig. 2, all SIS tunneling conductance show low subgap conductance as well as sharp quasiparticle peaks. These results suggest that $\mathrm{Bi}-2212$ could be one of the appealing candidates for superconducting devices e.g. SIS mixer.[16]

Most of our SIS junctions exhibited a Josephson current. The Ambegaokar-Baratoff (A-B) theory[17] for BCS superconductor predicts the relation

$$
I_{c} R_{n}=\frac{\pi \Delta(0)}{2 e}
$$

where $I_{c}$ is the Josephson current, $R_{n}$ is the resistance of the junction, and $\Delta(0)$ is the energy gap at $0 \mathrm{~K}$. Table I shows that as $\Delta(0)$ increases in the underdoped phase, the maximum $I_{c} R_{n}$ increases also. This links the quasiparticle gap to a superconducting energy scale. Furthermore,

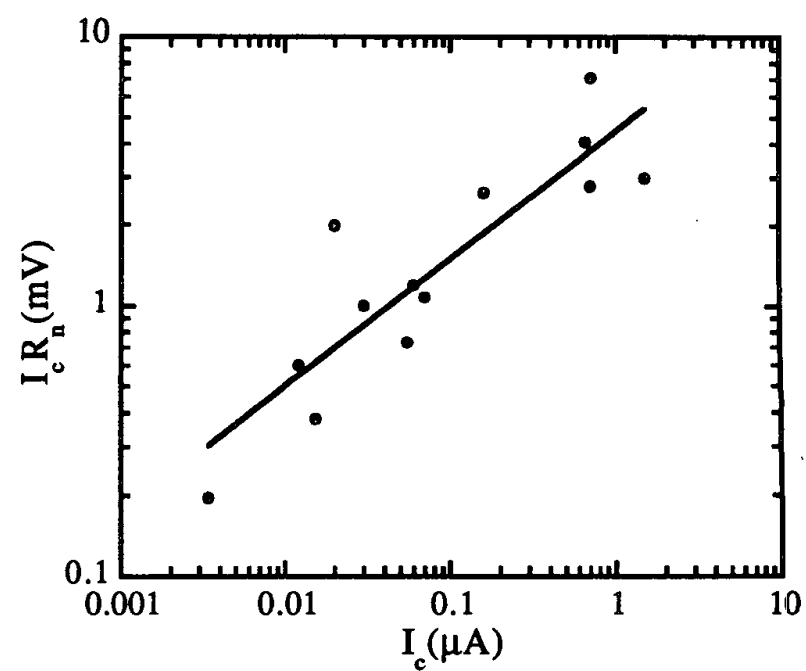

Fig. 3. $I_{c} R_{n}$ versus $I_{c}$ for overdoped Bi-2212 with $T_{c}=62 \mathrm{~K}$. The solid line corresponds to a power fit.

TABLE I

Doping Dependence of $I_{c} R_{n}$ ANd Energy Gap

\begin{tabular}{lccl}
\hline & $\begin{array}{c}\text { average } I_{c} R_{n}(\mathrm{mV}) \\
\text { (\# of Junc.) }\end{array}$ & $\begin{array}{c}\text { Max. } I_{c} R_{n} \\
(\mathrm{mV})\end{array}$ & $\Delta_{0}(\mathrm{meV})$ \\
\hline $\begin{array}{l}\text { overdoped } \\
\left(\mathrm{T}_{c}=62 \mathrm{~K}\right)\end{array}$ & $2.4(10)$ & 7.0 & $15-20$ \\
$\begin{array}{l}\text { optimally-doped } \\
\left(\mathrm{T}_{c}=92-95 \mathrm{~K}\right)\end{array}$ & $2.9(14)$ & 7.8 & $30-40$ \\
$\begin{array}{l}\text { underdoped } \\
\left(\mathrm{T}_{c}=83 \mathrm{~K}\right)\end{array}$ & $-(3)$ & 25 & $44-45$ \\
$\begin{array}{l}\text { underdoped } \\
\left(\mathrm{T}_{c}=74 \mathrm{~K}\right)\end{array}$ & $-(3)$ & 9.1 & $53-56$ \\
$\begin{array}{l}\text { underdoped } \\
\left(\mathrm{T}_{c}=70 \mathrm{~K}\right)\end{array}$ & $-(6)$ & 14.0 & $55-60$ \\
\hline
\end{tabular}

for overdoped Bi-2212[18] with $\mathrm{T}_{c}=62 \mathrm{~K}$, our data show an additional relationship between the Josephson current $I_{c}$ and the junction resistance $R_{n}$, where $R_{n}$ ranges from $1 \mathrm{k} \Omega$ to $100 \mathrm{k} \Omega$. There is a scaling behavior that $I_{\mathrm{c}} R_{n}$ is proportional to $\left(I_{c}\right)^{p}$ where $p=0.47$ as shown in Fig. 3 . This scaling behavior $(p \sim 0.5)$ has also been observed in tunneling in the $a-b$ plane for planar, ramp-edge, bicrystal grain boundary (GB), biepitaxial GB, and step-edge GB junctions.[4] The universality of $I_{c} R_{n}$ vs. $I_{c}$ allows us to estimate tunnel junction area in our break junctions, because the areas in grain boundary junctions are known. When we compare Fig. 3 with Fig. 1 of Ref.[4], the results lead to a junction area $\sim 500 \AA$ on edge.

There is an ongoing debate about the origin of the gap in tunneling measurements of HTS. Are the energy gaps seen in the tunneling data of Fig. 2 superconducting gaps, or are they of other origin? Markiewicz et al. [19] have proposed the idea that the gaps seen in tunneling measurement are charge density wave (CDW) gaps, or a mixed state of superconducting-CDW gap. To address this issue, we note that the Josephson current is observed simultaneously with the gap in the SIS conductance data. As shown in Table $I$, the average $I_{c} R_{n}$ and the maximum $I_{c} R_{n}$ values increase with increasing gap size. The maximum $I_{c} R_{n}$ value is $\sim 40 \%$ of $\Delta / e$ at each doping level, with a value as 
high as $25 \mathrm{mV}$ in underdoped case. The table shows only junctions with smaller than $30 \mathrm{k} \Omega$ junction resistances. The fact that the $I_{c} R_{n}$ values increases with increasing $\Delta(0)$ presents a strong case that the gaps observed in our tunneling data are superconducting energy gaps.

In summary, we investigated break junction tunneling measurements on $\mathrm{Bi}-2212$ with various doping levels. Simultaneous quasiparticle and Josephson tunneling current were observed, with $I_{c} R_{n}$ values as high as $25 \mathrm{mV}$ in one of the underdoped samples. It is observed that as the doping level decreases from overdoped to underdoped, the size of the superconducting energy gap increases. Moreover, as the size of the superconducting gap increase, the value of $I_{c} R_{n}$ also increases.

\section{REFERENCES}

[1] For a review see, ed. by $H$. Weinstock and $R$. Ralston, The New Superconducing Electronics Kluwer Academic Publishers, London (1993)

[2] K. E. Gray, "Tunneling in high-temperature superconductors," Mod. Phys. Lett. , vol. 2, pp. 1125-1130, 1988.

[3] K. Schlenga, R. Kleiner, G. Hechtfischer, M. Moble, S. Schmitt, P. Muller, C. Helm, C. Preis, F. Forsthofer, J. Keller, H. L. Johnson, M. Veith and E. Steinbeib, "Tunneling spectroscopy with intrinsic Josephson junctions in $\mathrm{Bi}_{2} \mathrm{Sr}_{2} \mathrm{CaCu}_{2} \mathrm{O}_{8+\delta}$ and $\mathrm{Tl}_{2} \mathrm{Ba}_{2} \mathrm{CaCu}_{2} \mathrm{O}_{x}, "$ Phys. Rev. $B$, vol. 57, pp. 14518-14536, June 1998.

[4] For a review see, R. Gross, L. Alf, A. Beck, O. M. Froehlich D. Koelle, A. Marx, "Physics and technology of high temperature superconducting Josephson junctions," IEEE Trans. Appl. Supercond. vol. 7, pp. 2929-2935, June 1997.

[5] Y. DeWilde, N. Miyakawa, P. Guptasarma, M. Iavarone, L. Ozyuzer, J. F. Zasadzinski, P. Romano, D. G. Hinks, C. Kendziora, G. W. Crabtree and K. E. Gray, "Unusual strong coupling effects in the tunneling spectroscopy of optimally doped and overdoped $\mathrm{Bi}_{2} \mathrm{Sr}_{2} \mathrm{CaCu}_{2} \mathrm{O}_{8+\delta}$, Phys. Rev. Lett. vol. 80, pp. 153-157, January 1998.

[6] C. Kendziora, R. J. Kelley, E. Skelton, M. Onellion, "Advances in single-crystal $\mathrm{Bi}_{2} \mathrm{Sr}_{2} \mathrm{CaCu}_{2} \mathrm{O}_{8}+\delta$ superconductors," Physica $C$ vol. 257, pp. 74-78, 1996.

[7] N. Miyakawa, P. Guptasarma, J. F. Zasadzinski, D. G. Hinks and K. E. Gray, "Strong dependence of the superconducting gap on oxygen doping from tunneling measurements on $\mathrm{Bi}_{2} \mathrm{Sr}_{2} \mathrm{CaCu}_{2} \mathrm{O}_{8+\delta}$," Phys. Rev. Lett. vol. 80, pp. 157-160, January 1998.

[8] L. Ozyuzer, J. F. Zasadzinski and K. E. Gray, "Point contact tunnelling apparatus with temperature and magnetic field control" Cryogenics, vol. 38, pp. 911-915, October 1998.

[9] E. L. Wolf, Principles of Electron Tunneling Spectroscopy, Oxford Univ. Press, New York, 1985.

[10] L. Ozyuzer, J. F. Zasadzinski, C. Kendziora and K. E. Gray, "Tunneling spectroscopy of overdoped $\mathrm{Bi}_{2} \mathrm{Sr}_{2} \mathrm{CaCu}_{2} \mathrm{O}_{8+\delta}$ single crystals," unpublished.

[11] M. R. Norman, H. Ding, J. C. Campuzano, T. Takeuchi, M. Randeria, T. Yokoya, T. Takahashi, T. Mochiku, K. Kadowaki, "Unusual dispersion and line shape of the superconducting state spectra of $\mathrm{Bi}_{2} \mathrm{Sr}_{2} \mathrm{CaCu}_{2} \mathrm{O}_{8+\delta}, "$ Phys. Rev. Lett. vol. 79, pp. 3506-3509, November 1997; Z. X. Shen, P. J. White, D. L. Feng, C. Kim, G. D. Gu, H. Ikeda, R. Yoshizaki, and N. Koshizuka, "Temperature-induced momentum dependent spectral weight transfer in $\mathrm{Bi}_{2} \mathrm{Sr}_{2} \mathrm{CaCu}_{2} \mathrm{O}_{8}+\delta$," Science vol. 280, pp. 259-262, April 1998.

[12] J. R. Kirtley, C. C. Tsuei, J. Z. Sun, C. C. Chi, L. S. YuJahnes, A. Gupta, M. Rupp, and M. B. Ketchen, "Symmetry of the order parameter in the high $T_{c}$ superconductor $\mathrm{YBa}_{2} \mathrm{Cu}_{3} \mathrm{O}_{7-x}$," Nature vol. 373, pp. 225-228, January 1995.

[13] $H$. Won and $K$. Maki, " $d$-wave superconductor as a model of

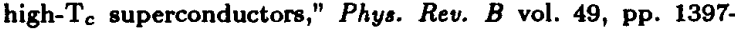
1402, January 1994.

[14] Z. Yusof, J. F. Zasadzinski, L. Coffey, N. Miyakawa, "Modeling of tunneling spectroscopy in high- $T_{c}$ superconductors incorporating band structure, gap symmetry, group velocity, and tunneling directionality," Phys. Rev. B vol. 58, pp. 514-521, July 1998.

[15] J. Halbritter, "Pseudo gap, resonant tunneling and charge dynamics in cuprate superconductors," Physica $C$ vol. 302, pp. 221-228, 1998.

[16] K. Kouznetsov, L. Coffey, J. F. Zasadzinski, "Modeling the performance of high temperature superconductor based SIN and SIS quasiparticle mixers," Applied Superconductivity vol. 2, pp. 735-740, 1994.

[17] V. Ambegaokar and A. Baratoff, "Tunneling between superconductors," Phys. Rev. Lett. vol. 10, pp. 486-489, June 1963.

[18] We could not be successfull to observe Josephson current in a large range of $R_{n}$ for optimally-doped and underdoped crystals.

[19] R. S. Markiewics, C. Kusko, and M. T. Vaughn, "Tunneling and photoemission in an SO(6) superconductor," Journal of Supereonductivity, in press. 